\title{
Beamforming Degradation of a W-CDMA Smart Antenna Under FDD and MAI Channel Estimation Errors
}

\author{
Chi-Min Li, Hsueh-Jyh Li, Senior Member, IEEE, and Pao-Jen Wang
}

\begin{abstract}
A smart antenna adopts the beamforming technique to increase the signal-to-(interference plus noise) ratio (SINR) and system capacity. In this paper, the impact of two channel estimation errors-frequency-division duplex (FDD) estimation error and multiple-access interference (MAI) estimation error for the beamforming of a wide-band code-division multiple-access smart antenna-are analyzed by using the measured channel state information. Results show that for a complex conjugate beamforming, FDD estimation error will cause median degradation of less than $1 \mathrm{~dB}$ if the desired signal is in a line-of-sight (LOS) channel and 1-3 dB if in a non-LOS (NLOS) channel. In addition, the MAI estimation error will cause 0.3-0.5 dB degradation per interference in the $\mathrm{LOS}$ case and $0.5-1 \mathrm{~dB}$ degradation in the NLOS case.
\end{abstract}

Index Terms-Line-of-sight (LOS), non-LOS (NLOS), smart antenna, wide-band code-division multiple access (W-CDMA).

\section{INTRODUCTION}

W IDE-BAND code-division multiple-access (W-CDMA) systems have been widely commercialized recently due to their high system capability. A W-CDMA system can deploy the smart antenna receiver at the base station (BS) to increase the received signal-to-noise ratio (SNR). A smart antenna forms a main beam to the desired user and nulls the undesired ones with the help of proper weighting sets estimated by the pilot bits on the dedicated physical control channel (DPCCH) [1]. Nevertheless, if the channel attenuation is not perfectly estimated during signal transmission, the output signal power of the smart antenna will be degraded [6].

Most research in the literature has focused on studying the performance on different beamforming methods theoretically and tried to establish relations between uplink and downlink channel for the W-CDMA system [2]-[5]. In [2], direction-ofarrival and complex conjugate (CC) methods are compared to simulate their uplink and downlink SNR performance. In [5],

Manuscript received September 15, 2006; revised January 30, 2007. This work was supported by the National Science Council, R.O.C., under Grant NSC 95-2221-E-019-018.

The authors are with the Department of Communication and Guide Engineering, National Taiwan Ocean University, Keelung 202-24, Taiwan, R.O.C. and with the Graduate Institute of Communication Engineering, National Taiwan University, Taipei 106-17, Taiwan, R.O.C.

Color versions of one or more of the figures in this paper are available online at http://ieeexplore.ieee.org.

Digital Object Identifier 10.1109/LAWP.2007.893100

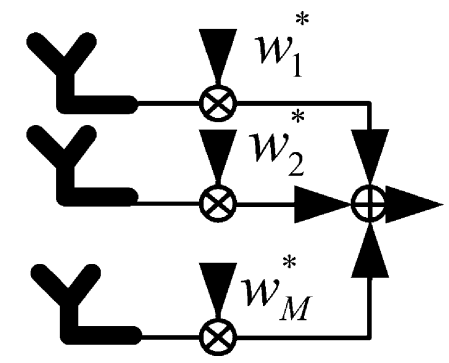

Fig. 1. A smart antenna architecture.

two methods, null constrain and frequency calibration, were proposed to execute the downlink beamforming by modifying uplink weightings. To the best of the authors' knowledge, few field measured results were reported on such beamforming issues when channel estimation errors exist. In this paper, we evaluate the impact on beamforming of a smart antenna receiver for two different channel estimation errors: the frequency-division duplex (FDD) operation and multiple-access interference (MAI).

In the FDD mode, a BS with smart antennas uses the uplink channel estimation to the downlink beamforming. Channel estimation errors occur since the downlink and uplink channels use different frequency bands for transmission. In addition, in the time-division duplex (TDD) mode, channel estimation errors also exist because the BS has to serve multiple users at the same time, i.e., MAI problems occur. This paper analyzes these errors by using the measured channel state information (CSI). In Section II, we briefly describe the degradation problem of a smart antenna under the FDD and MAI environments. In Section III, experiments with FDD and MAI channel estimation errors are carried out in two different channels: line-of-sight (LOS) and non-LOS (NLOS). Some conclusions of this paper are given in Section IV.

\section{Signal Degradation}

A conventional smart antenna can be depicted in Fig. 1. Assume there are $M$ antenna elements at the receiving site; each element can multiply an appropriate weighting $w_{m}^{*}, m=1 \ldots M$ to compensate the channel distortion. With these weightings, smart antennas can coherently sum up these signals to increase output SNR. 

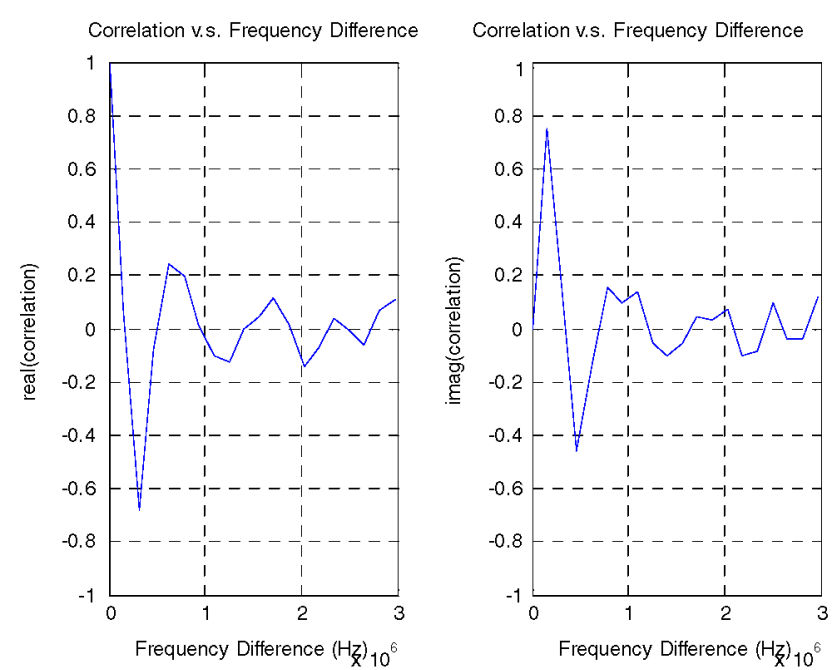

Fig. 2. Correlation versus frequency difference (LOS).

\section{A. FDD Estimation Error}

Assume that a $P$-paths uplink time-invariant channel for the $k$ th user at the $m$ th receiving antenna can be modeled as

$$
U_{m, k}(t)=\sum_{p=0}^{P-1} U_{p, m, k} \delta\left(t-\tau_{p, m, k}\right)
$$

where $U_{p, m, k}$ is the complex uplink channel attenuation at time $\tau_{p, m, k} . \tau_{p, m, k}$ is the time-of-arrival (TOA) of the $p$ th multipath at the $m$ th receiving antenna for the $k$ th user. If the instantaneous downlink channel for the $k$ th user has the same TOAs but with different attenuations compared to the uplink channel, the corresponding downlink channel model for the $k$ th user can be expressed as

$$
D_{m, k}(t)=\sum_{p=0}^{P-1} D_{p, m, k} \delta\left(t-\tau_{p, m, k}\right)
$$

where $D_{p, m, k}$ is the complex downlink attenuation. In FDD, $U_{p, m, k}$ and $D_{p, m, k}$ could be very different due to the adoption of different frequency bands. If we define the normalized frequency correlation coefficient as

$$
\rho(\Delta k)=\frac{H(k) H^{*}(k+\Delta k)}{\Omega}
$$

where $H(k)$ is the channel frequency response, $\Omega=$ $E\left\{|H(k)|^{2}\right\}, \Delta k$ is the frequency difference, and $E\{\cdot\}$ is the expectation operator. Two correlation examples of the measured outdoor LOS and NLOS paths are shown in Figs. 2 and 3. Both examples illustrate the correlation variation defined in (3) versus frequency difference between the uplink and downlink channels. Results show that for a given correlation coefficient (take $\rho=0.5$ as an example), coherent bandwidth for the outdoor environment is approximately less than $200 \mathrm{KHz}$ for both cases. That is, for uplink and downlink channels with frequency difference greater than $200 \mathrm{KHz}$, they can be treated as uncorrelated ones. Although Figs. 2 and 3 are only two
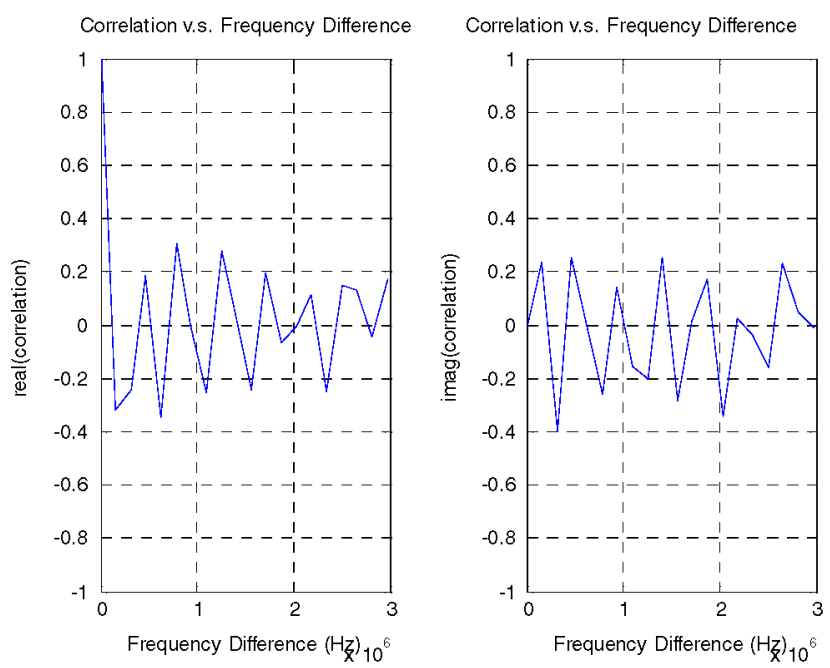

Fig. 3. Correlation versus frequency difference (NLOS).

examples, they reveal that $U_{p, m, k}$ and $D_{p, m, k}$ will be quite different in a typical $190 \mathrm{MHz}$ frequency separation for the 3GPP FDD uplink and downlink channels [1].

Assume each multipath can be compensated with a proper delay $\tau_{p, m, k}$ by using a RAKE receiver and let $P=1$ and the zeroth user be the desired user. The estimated weightings of the uplink beamforming at $\tau_{1, m, 0}$ by using the $\mathrm{CC}$ beamforming technique [2] are

$$
\vec{U}_{0}=\frac{1}{S}\left[\begin{array}{c}
U_{1,1,0}^{*} \\
U_{1,2,0}^{*} \\
\vdots \\
U_{1, M, 0}^{*}
\end{array}\right]
$$

where $S=\sqrt{\sum_{m=1}^{M}\left|U_{1, m, 0}\right|^{2}}$ is the normalization factor for power and "** denotes the complex conjugate. However, if perfect channel estimation is achievable, the downlink beamforming weightings should be

$$
\vec{D}_{0}=\frac{1}{T}\left[\begin{array}{c}
D_{1,1,0}^{*} \\
D_{1,2,0}^{*} \\
\vdots \\
D_{1, M, 0}^{*}
\end{array}\right]
$$

where $T=\sqrt{\sum_{m=1}^{M}\left|D_{1, m, 0}\right|^{2}}$. Therefore, if BS uses the channel estimation obtained from uplink channel to the downlink beamforming, the output signal of the smart antenna will suffer SNR degradation and can be evaluated as

$$
\begin{aligned}
\text { Degradation }_{\mathrm{dB}} & =20 \log \left(\frac{\vec{D}_{0}^{H} \vec{U}_{0}}{\vec{D}_{0}^{H} \vec{D}_{0}}\right) \\
& =20 \log \left(\vec{D}_{0}^{H} \vec{U}_{0}\right)
\end{aligned}
$$

where $H$ denotes the Hermitian operation.

\section{B. MAI Estimation Error}

Next, consider a multiple access environment with MAI estimation errors and assume there are $K+1$ active users served 


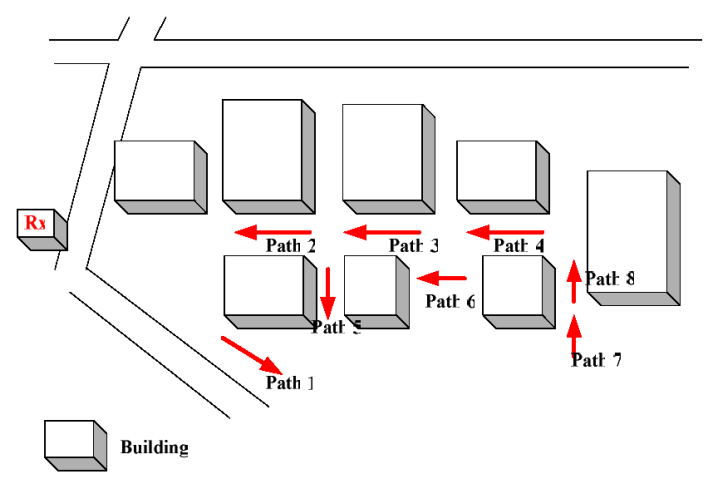

Fig. 4. Environment of measurement.

by the same BS. The uplink channel model for the BS can be described as

$$
\begin{aligned}
V_{m}(t) & =\sum_{k=0}^{K} U_{m, k}(t) \\
& =\sum_{k=0}^{K} \sum_{p=0}^{P-1} U_{p, m, k} \delta\left(t-\tau_{p, m, k}\right) .
\end{aligned}
$$

Due to the MAI interferences, the estimated uplink weightings for the desired user at $\tau_{1, m, 0}$ are

$$
\vec{V}_{0}=\frac{1}{S^{\prime}}\left[\begin{array}{c}
U_{1,1,0}^{\prime *} \\
U_{1,2,0}^{\prime *} \\
\vdots \\
U_{1, M, 0}^{\prime *}
\end{array}\right]
$$

where $S^{\prime}=\sqrt{\sum_{m=1}^{M}\left|U_{1, m, 0}^{\prime}\right|^{2}}, U^{\prime}=U+\Delta U$ and $\Delta U$ is the estimation error. Hence, the degradation can be evaluated as

$$
\begin{aligned}
\text { Degradation }_{\mathrm{dB}} & =20 \log \left(\frac{\vec{V}_{0}^{H} \vec{U}_{0}}{\vec{U}_{0}^{H} \vec{U}_{0}}\right) \\
& =20 \log \left(\vec{V}_{0}^{H} \vec{U}_{0}\right)
\end{aligned}
$$

where $\vec{U}_{0}$ is defined in (4).

\section{EXPERIMENT RESULTS}

In this section, some field measured CSIs are tested to evaluate the degradations defined in (6) and (9) for the FDD and MAI channel estimation errors. CSIs were measured by using the RUSK channel sounder. ${ }^{1}$ The measuring instrument contains one vertical polarized transmitting omnidirectional antenna and eight vertically polarized receiving antennas. Spacing between the receiving antenna elements is $\lambda / 2$. $\lambda$ is the wavelength, and the carrier frequency is $2.44 \mathrm{GHz}$. Total measuring bandwidth is $120 \mathrm{MHz}$. Fig. 4 shows the layout of the experiment. The antenna array receiver was placed on the top of an 11-floor building and was marked as Rx in Fig. 4.

In this experiment, both LOS and NLOS cases are evaluated for comparisons. Paths 1-4 are the LOS cases and Paths 5-8 are the NLOS cases. In addition, to satisfy the W-CDMA require-

\footnotetext{
${ }^{1}$ http://www.channelsounder.de/index.php.
}

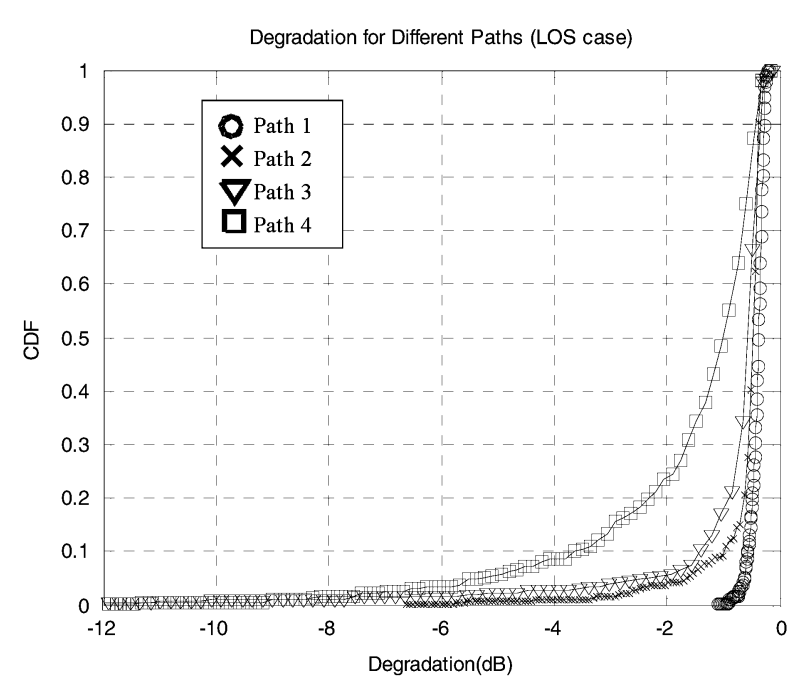

Fig. 5. FDD degradation of LOS paths.

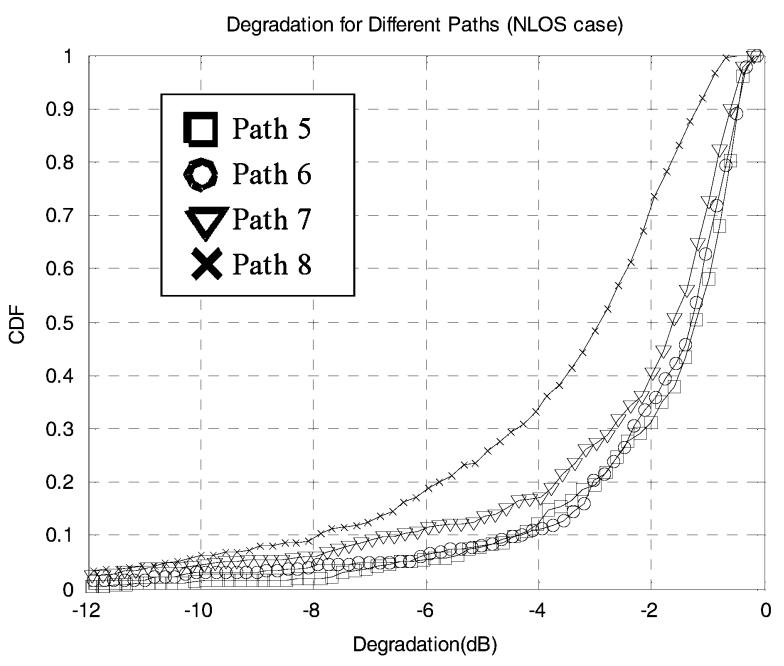

Fig. 6. FDD degradation of NLOS paths.

ment $(5 \mathrm{MHz}$ signal bandwidth and $190 \mathrm{MHz}$ FDD uplink and downlink channel frequency separation), we use the $2380-2385 \mathrm{MHz}$ for the uplink channel and $2495-2500 \mathrm{MHz}$ for the downlink channel. The frequency separation of the uplink and downlink channel is $110 \mathrm{MHz}$ in this experiment. Four hundred delay profiles along each path are chosen for the statistical analysis. The typical length of each path is about $5 \mathrm{~m}$. The spreading factor of the DPCCH is set to 256 for all users. Figs. 5 and 6 are the cumulative distribution function results of signal degradation for the LOS and NLOS cases. We can note that signal has less degradation in the LOS case (the median degradation is less than $1 \mathrm{~dB}$ ) than in the NLOS case (the median degradation is from $1 \mathrm{~dB}$ to $3 \mathrm{~dB}$ ). The degradation increases as the distance between $T x-R x$ increases.

To evaluate the impact of MAI interferences, we synthesized the MAI scenario as follows: the MAI users were randomly distributed along Paths 1,5, and 8. That is, interferences may have LOS or NLOS paths to the receiver. The desired user was moving along Path 2 for the LOS case and Path 6 for the NOLS cases. The number of MAI interference sources is chosen from one to nine. Figs. 7 and 8 are the results if we compared the 


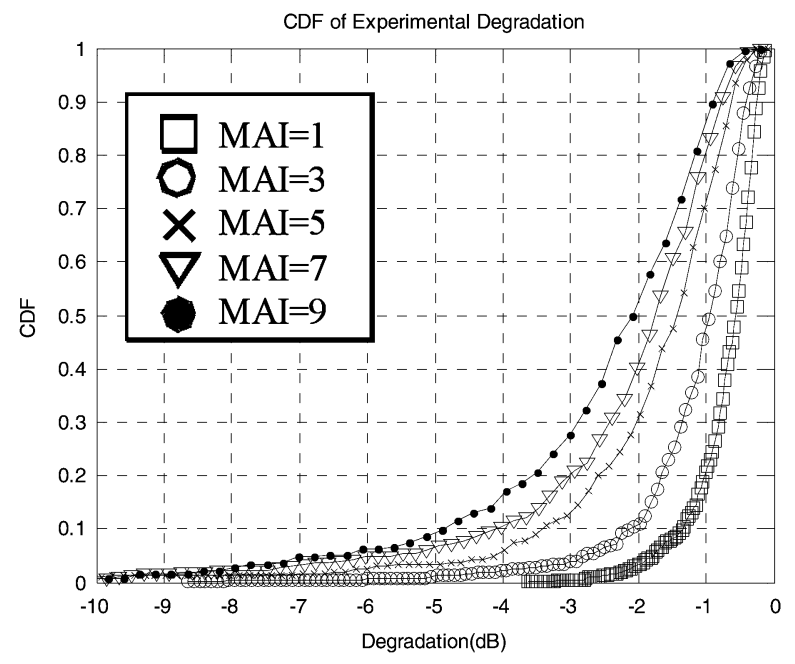

Fig. 7. MAI degradation of LOS paths.

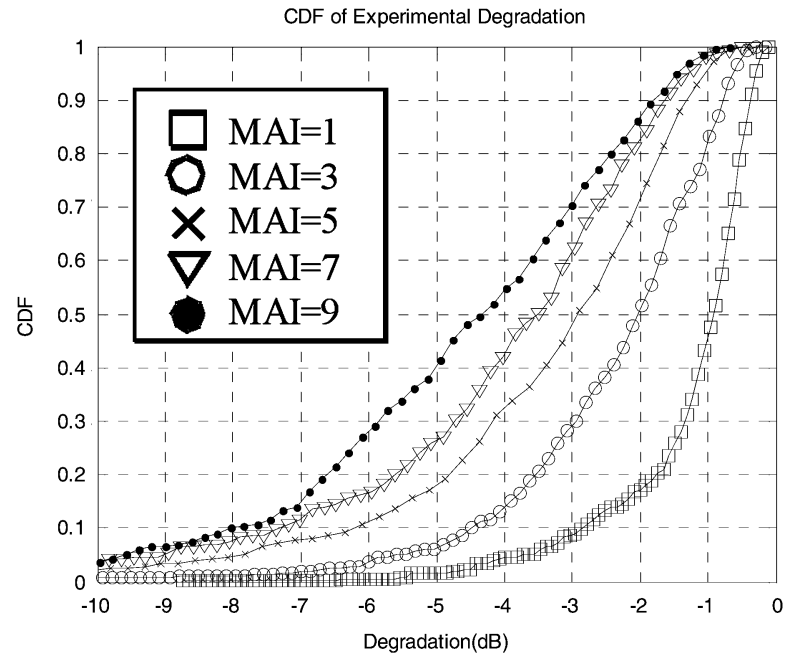

Fig. 8. MAI degradation of NLOS paths. beamforming degradation in an MAI scenario for the LOS and NLOS cases, respectively. The same phenomenon occurs compared with the FDD estimation error, i.e., LOS channels will have less SNR degradation than the NLOS cases. A rule-ofthumb result reveals from the experiments that the smart antenna will suffer $0.3-0.5 \mathrm{~dB}$ SNR degradation per interference if the desired user is in the LOS channel and $0.5-1 \mathrm{~dB}$ degradation in the NLOS case.

\section{CONCLUSION}

In this paper, the beamforming degradations of a smart antenna from the FDD and MAI estimation errors are investigated. Results show that FDD estimation error will cause median degradation less than $1 \mathrm{~dB}$ if the desired signal is in the LOS channel and $1-3 \mathrm{~dB}$ if in the NLOS channel. In addition, the MAI interference will cause $0.3-0.5 \mathrm{~dB}$ degradation per interference for the LOS channel and $0.5-1 \mathrm{~dB}$ degradation for the NLOS channel. These rule-of-thumb results can help system engineers to predict the received signal quality of a W-CDMA smart antenna system if the FDD and MAI channel estimation errors exist.

\section{REFERENCES}

[1] 3rd Generation Partnership Project (3GPP), Spreading and modulation (FDD) Tech. Rep. TS 25.213 V2.0.0, 1999.

[2] H.-J. Li and T.-Y. Liu, "Comparison of beamforming techniques for W-CDMA communication systems," IEEE Trans. Veh. Technol., vol. 52, pp. 752-760, Jul. 2003.

[3] B. K. Chalise, L. Haring, and A. Czylwik, "Uplink to downlink spatial covariance matrix transformation concepts for downlink beamforming," in Proc. IEEE Int. Symp. Signal Process. Inf. Technol., Dec. 2003, pp. 568-571.

[4] A. Arredondo and G. Xu, "Vector channel modeling and prediction for downlink beamforming in frequency-division duplex systems," IEEE Trans. Commun., vol. 50, pp. 1571-1576, Oct. 2002.

[5] Y.-C. Liang, "FDD DS-CDMA downlink beamforming by modifying uplink beamforming weights," in Proc. Veh. Technol. Conf., Sep. 2000, vol. 1, pp. 170-174

[6] J. C. Liberti and T. S. Rappaport, Smart Antennas for Wireless Communications. Englewood Cliffs, NJ: Prentice-Hall, 1999. 